\title{
Effect of Combining Infrared Heating with Ultraviolet Irradiation on Inactivation
}

\section{of Mold Spores}

\author{
Daisuke HamanakA $^{1 *}$, Griffiths Gregory Atungulu ${ }^{2}$, Fumihiko TAnaKa ${ }^{1}$ and Toshitaka Uchino ${ }^{1}$ \\ ${ }^{1}$ Faculty of Agriculture, Kyushu University,6-10-1, Hakozaki, Higashi-ku Fukuoka 812-8581, Japan \\ ${ }^{2}$ Department of Biological and Agricultural Engineering, University of California, One Shields Avenue Davis, CA 95616, USA
}

Received November 5, 2009; Accepted March 17, 2010

Combining effect of infrared radiation (IR) heating with ultraviolet (UV) irradiation on the inactivation of mold spores isolated from citrus fruits was investigated. Fruit-related molds (Penicillium, Aspergillus, Cladosporium, Rhizopus, and Byssochlamys) were inoculated on stainless steel Petri dish and inactivated by IR heating, UV irradiation, and combined treatments with different sequence orders. Three isolates (Penicillium, Aspergillus and Cladosporium) were sensitive to IR heating, while Rhizopus and Byssochlamys spores were relatively sensitive to UV irradiation rather than IR heating. Combinations of IR heating with UV irradiation were effective in the inactivation of all mold spores. Considerable reductions in the survival of all mold spores were achievable by the combined treatment regardless of the treatment order.

Keywords: infrared radiation heating, ultraviolet irradiation, mold spores, combined treatment

\section{Introduction}

Fungal contamination is one of the most serious problems during storage of various fruits (Tournas and Katsoudas, 2005). Fruits which have relatively stronger peels, such as citrus fruits, exhibit fungal contamination during long-term storage. However, in the case of fruits with weak peels, such as strawberry, grape, and fig, severe fungal proliferations were observed within a few days after storage. The change in appearance, off-odor and mycotoxin production resulting from fungal contamination are serious problems during the postharvest stage. Prevention of these fungal contaminations is therefore essential and can be achieved using optimum decontaminating techniques.

Chemicals (gas or agent) are the most commonly employed decontaminants in the antimicrobial processing of fruits (Zhang, 2007; Song et al., 2007; Zheng et al., 2007). However, recently, consumer preference and concerns over environmental safety have placed pressure on fruit and vegetable processors to seek alternative methods for sustainable decontamination of fruits and vegetables.

In our previous study, we applied infrared radiation (IR)

*To whom correspondence should be addressed.

E-mail: hamanaka@bpes.kyushu-u.ac.jp heating to achieve surface decontamination of citrus fruits (Uchino et al., 2004), although discoloration and softening of the fruits' surfaces due to penetration of thermal energy during IR heating were sometimes observed. Therefore, in order to reduce the thermal effect on the fruits' surfaces without lessening the decontamination ability, we explored the effect of decreasing the input energy of IR heating by combining it with other non-thermal techniques.

The microbiocidal effect of ultraviolet (UV) irradiation with a wavelength band of UV-C (100-280 nm) is widely known, and has been applied for decontamination in various industrial fields. Regarding prevention of postharvest decay in fruits, many researchers have tested the decontaminating effect of UV on fruits, such as grape and strawberry, and have suggested that UV irradiation is effective in reducing the microbial population on fruits (Baka et al., 1999; Nigro et al., 1998). In addition, the killing effect of UV irradiation was accelerated by combining treatments with other antimicrobial techniques (Yano, 2001; Hadjok et al., 2008; Jung et al., 2008). To improve the decontamination efficiency of IR heating, combination with UV irradiation shows promise without reducing the quality of the fruit due to excessive treatment of IR heating. On the other hand, there were some disadvantages in the UV decontaminating procedure, 
including the limited effect on the material surface only and minimal effect on some kinds of mold spores. Nevertheless, no report has elucidated the effects of combining IR heating with UV irradiation on the improvement of the decontaminating efficiency of fruit and fruit-related microorganisms.

The aims of this study were to investigate the effect of combining IR heating with UV irradiation on the inactivation of some citrus fruit-related mold spores in vitro. The data obtained will be useful in designing devices for IR and UV decontamination systems for various kinds of fresh fruits.

\section{Materials and Methods}

Fruit materials and mold isolation Wild-type molds isolated from citrus fruit were used in this study. The isolating procedure was as follows. A 10-g peel from a fruit sample (cv. Citrus unshiu) cultivated and harvested in Fukuoka City, Japan, was macerated by a stomacher (BagMixer, Interscience, Saint Nom La Bretèche, France) with $90 \mathrm{~mL}$ of sterile distilled water for $5 \mathrm{~min}$. The peel debris was filtrated by a sterile stomacher bag, and the filtrate was used as the microbial suspension. The microbial suspensions were spread on a potato dextrose agar (PDA; Nissui Pharmaceutical Co., Ltd., Tokyo, Japan) plate, and incubated at $25^{\circ} \mathrm{C}$ for $48 \mathrm{~h}$. After colonization, the resulting colonies were isolated without contamination, and re-cultured under the same conditions. Thus, five molds, of which colors and morphological properties were different, were isolated and their genuses identified (Lee, 2005; Aihara and Lee, 2005; Kosuge and Ota, 2005). These five molds were used in the following study.

Preparation of spore suspension Isolated molds pure grown on a PDA plate were harvested by the spreader after mixing with sterile distilled water containing $0.1 \% \mathrm{w} / \mathrm{v}$ surfactant (Tween 80, Wako Pure Chemical Co. Ltd., Tokyo, Japan). The harvested mycelia were eliminated by filtration using sterile glass wool. Mold spores in the filtrates were washed three times by centrifugation $(2500 \times \mathrm{g}, 10 \mathrm{~min})$ in sterile distilled water. The initial concentration of the mold spores was adjusted to approximately $10^{6}-10^{7} \mathrm{CFU} / \mathrm{mL}$. These prepared spore suspensions were stored at $4^{\circ} \mathrm{C}$ until use.

Sample preparation for inactivating treatment In order to investigate the characteristics of spore inactivation by single and sequential treatment of IR and UV, stainless steel Petri dishes $(19 \times \phi 90 \mathrm{~mm})$ were used as reported in our previous studies (Hamanaka et al., 2005, 2006). A 2-mL sample of the prepared spore suspensions was inoculated onto a stainless steel Petri dish, spread evenly, and air-dried on a clean bench (MCV-710ATS, Sanyo Electric Co., Ltd., Osaka, Japan) at room temperature. The air dried samples were placed in desiccator jars containing silica gel at $25^{\circ} \mathrm{C}$ for dry-
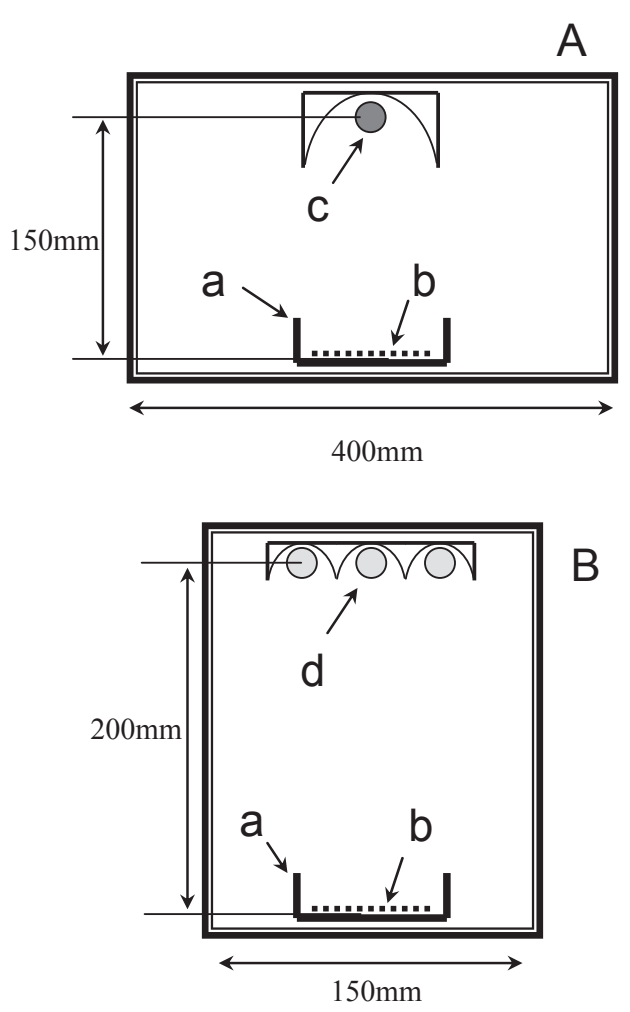

Fig. 1. Schematic diagrams of sterilizing chamber for IR heating (A) and UV irradiation (B). a: $\phi 90-\mathrm{mm}$ stainless steel Petri dish, b: mold ascospores, c: IR heater, d: UV lamp.

ing. After two days of desiccation, the sterilizing processes were conducted.

Disinfection procedure Figure 1 shows the experimental chambers. IR chamber was equipped with an IR heater $(220 \times \phi 10.5$ mm; IRE135-M, Iwasaki Electric Co., Ltd., Tokyo, Japan). The electric power of the heater was $0.5 \mathrm{~kW}$. The peak wavelength and energy of the heater was $950 \mathrm{~nm}$, $4.2 \mu \mathrm{W} / \mathrm{cm}^{2} / \mathrm{nm}$ at $10 \mathrm{~cm}$ below the emitter surface, respectively. The UV chamber was equipped with three UV lamps $(900 \times \phi 20.5 \mathrm{~mm}$; QGL100-2X, Iwasaki Electric). The wavelength and energy of the UV lamp were $254 \mathrm{~nm}$ and 20 $\mathrm{mW} / \mathrm{cm}^{2}$ at $10 \mathrm{~cm}$ below, respectively. The inner surface of both chambers was covered with an aluminum sheet to reflect IR and UV, and to prevent energy from leaking out of the chamber. All IR heating and UV irradiation experiments were carried out after ensuring the stability of irradiation from the IR heater and UV lamp (about $5 \mathrm{~min}$ ). A stainless steel Petri dish inoculated with spores was set $150 \mathrm{~mm}$ and $200 \mathrm{~mm}$ below the IR heater and UV lamp, respectively. Figure 2 shows the temperature profile of the stainless steel Petri dish $(\phi 90$ $\mathrm{mm}$ ) without samples, which was monitored by a T-type thermocouple attached to the surface of the stainless steel Petri dish. The treatment periods for IR heating and UV irradiation and their combination are shown in Table 1. In this study, the treatment periods for IR heating and UV irradiation were the 


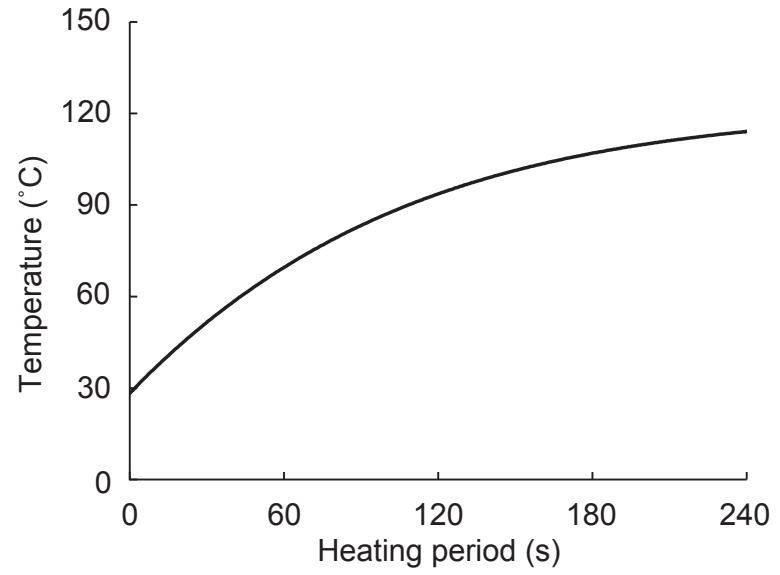

Fig. 2. Temperature profile on stainless steel Petri dish during IR heating. Temperature data were obtained by a T-type thermocouple attached to the surface of the stainless steel Petri dish.
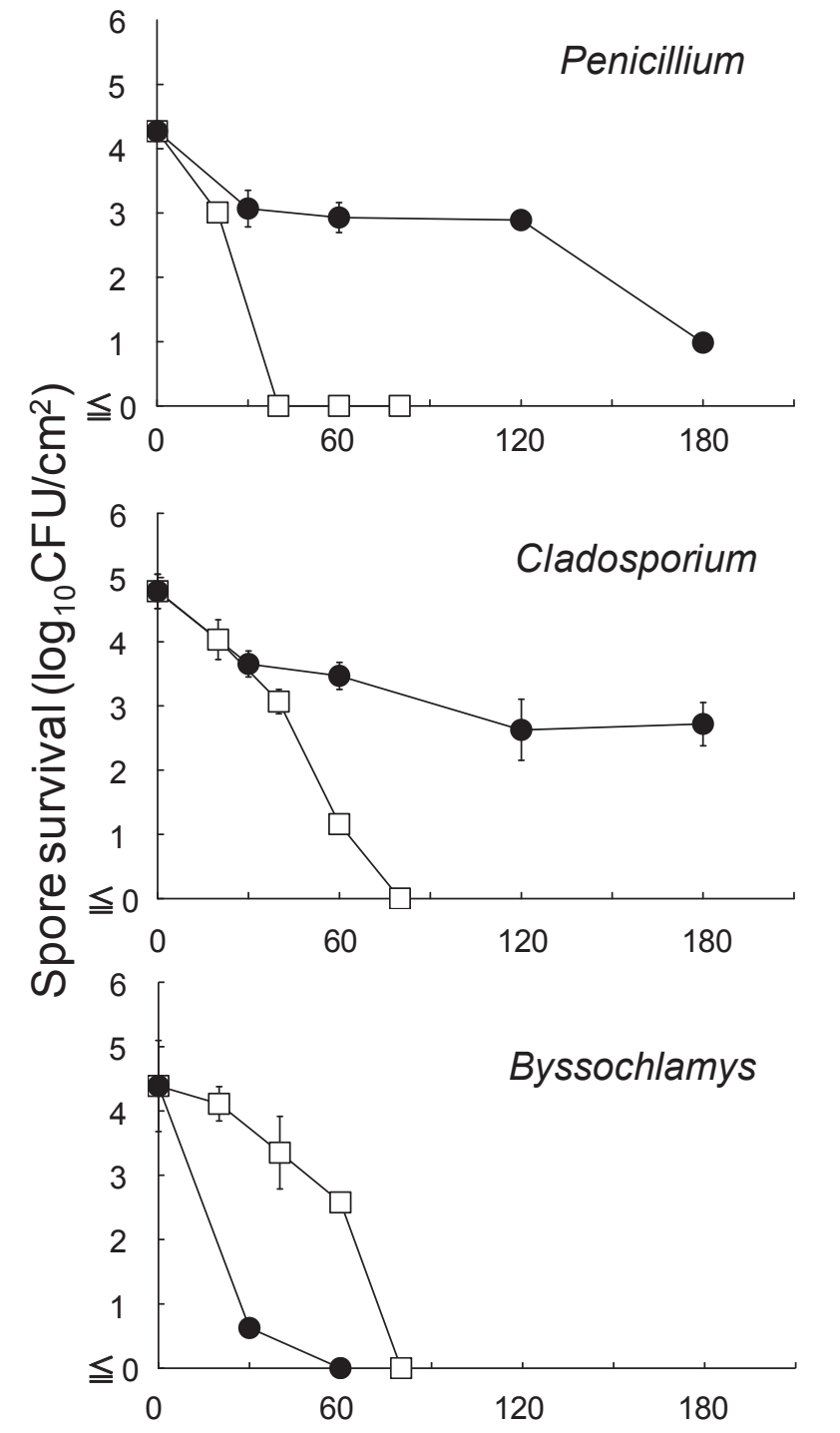

Treatment period of IR heating or UV irradiation (sec)

Fig. 3. Survival of five kinds of mold spores treated by IR heating, UV irradiation. Vertical bars indicate standard error ( $\mathrm{n}=5$ ) and are not shown when masked by the graph symbols. Used molds were morphologically and microscopically identified as Penicillium sp., Aspergillus sp., Cladosporium sp., Rhizopus sp., and Byssochlamys sp. $\square$ : IR heating, @: UV irradiation.

Table 1. Treatment periods (seconds) of IR heating, UV irradiation, and their combinations.

\begin{tabular}{|c|c|c|c|}
\hline IR & UV & $\mathrm{IR} \rightarrow \mathrm{UV}^{*}$ & $\mathrm{UV} \rightarrow \mathrm{IR}^{*}$ \\
\hline \multirow{4}{*}{$0-80$} & \multirow{4}{*}{$0-180$} & $5 \rightarrow 5$ & $5 \rightarrow 5$ \\
\hline & & $10 \rightarrow 10$ & $10 \rightarrow 10$ \\
\hline & & $20 \rightarrow 20$ & $20 \rightarrow 20$ \\
\hline & & $30 \rightarrow 30$ & $30 \rightarrow 30$ \\
\hline
\end{tabular}

$* I R \rightarrow$ UV $(\mathrm{UV} \rightarrow \mathrm{IR})$ means IR treatment followed by UV irradiation (UV irradiation followed by IR treatment). After IR treatment, samples were immediately cooled to around room temperature in a refrigerator for $2 \mathrm{~min}$.
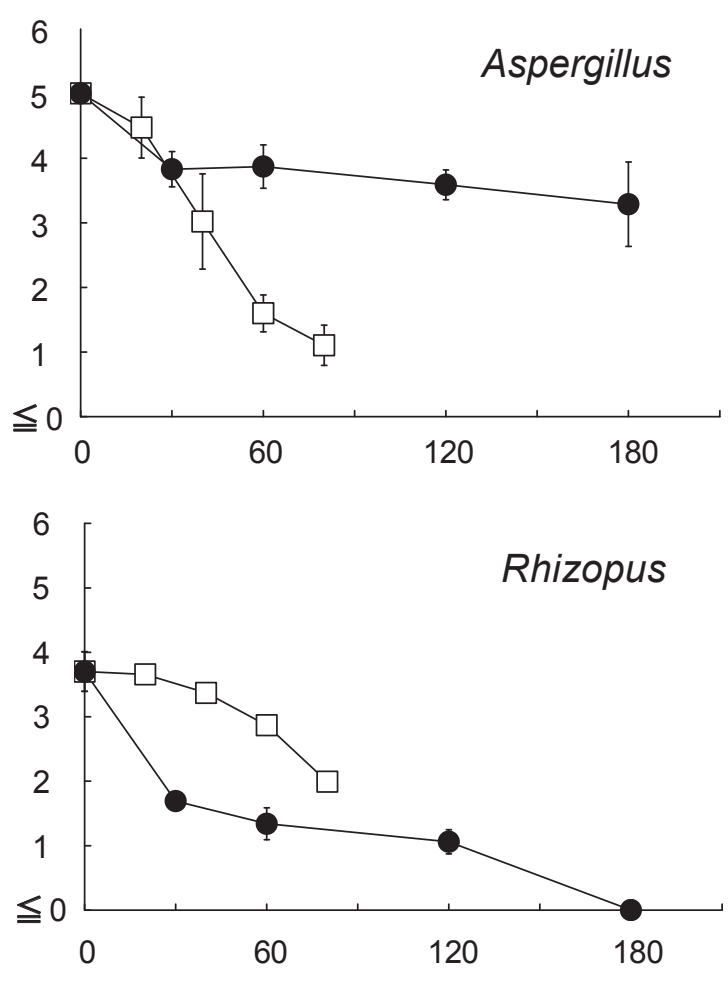


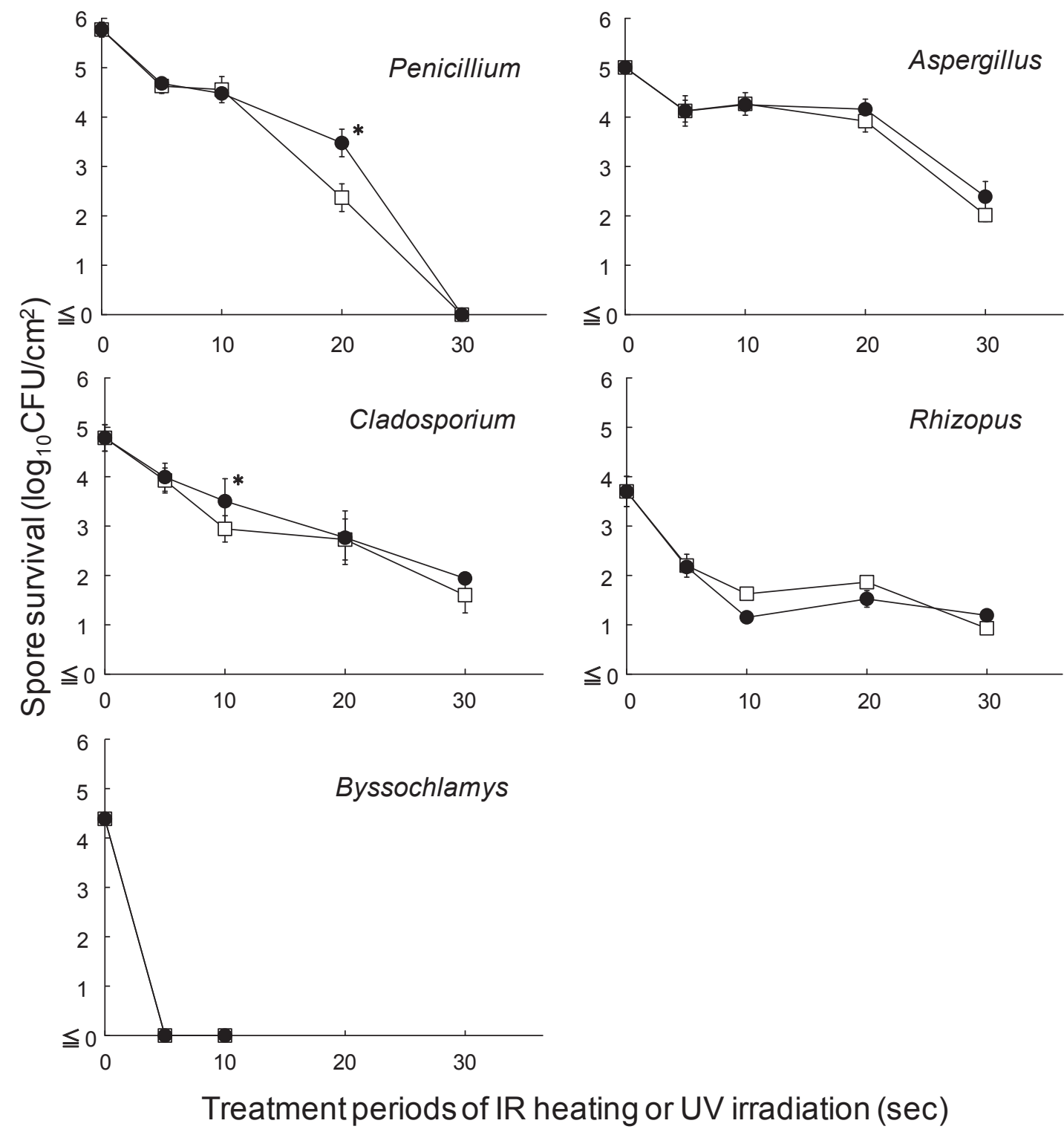

Fig. 4. Survival of five kinds of mold spores after sequential treatment of IR and UV. Vertical bars indicate standard error ( $\mathrm{n}=5$ ) and are not shown when masked by the graph symbols.

$\square$ : IR heating followed by UV irradiation, O: UV irradiation followed by IR heating. Horizontal axis means the treatment periods of IR heating or UV irradiation. (e.g., The plots of ' $30 \mathrm{sec'}$ at the graph symbol ' $\square$ ' indicates the treatment at 30 sec of IR heating followed by 30 sec of UV irradiation.) Asterisk (*) means the significant difference by Student's $t$-test $(\mathrm{p}<0.05)$.

same regardless of the treatment order. In the sequential treatment, the IR heated sample was cooled to room temperature in a refrigerator for $120 \mathrm{~s}$ before UV irradiation and then resuspended in sterile distilled water.

Mold counting A 50-mL aliquot of sterile distilled water was added to a Petri dish, and spores were removed from the stainless steel surface and re-suspended in sterile distilled water using ultrasonic treatment (2510J-MTH, Yamato Scientific Co., Ltd., Tokyo, Japan) for $3 \mathrm{~min}$. The amount of initial and surviving spores was determined on a PDA plate. These plates were incubated at $25^{\circ} \mathrm{C}$, and the colonies that had grown after $48 \mathrm{~h}$ were counted. In this study, the number of colonies after incubation was considered as the number of surviving spores. The inactivating effects of each trial were expressed as the number of surviving spores per unit area of the stainless steel Petri dish $\left(\log _{10} \mathrm{CFU} / \mathrm{cm}^{2}\right)$.

Statistical analysis All data for the number of surviving spores represent the mean of at least five experimental replicates. Significant differences in the data of combining treatments at the same treatment periods were determined by Student's $t$-test $(p<0.05)$.

\section{Results and Discussion}

The surfaces of citrus fruit can be contaminated with various species of molds (Tournas and Katsoudas, 2005). In this study, the five molds isolated from the surface of citrus 
fruits were identified as Penicillium, Aspergillus, Cladosporium, Rhizopus and Byssochlamys based on the color and morphological properties. The sensitivity among these molds to IR heating and UV irradiation differed as shown in Fig. 3. Among the isolates, three mold spores (Penicillium, Aspergillus, and Cladosporium) were more sensitive to IR heating than to UV irradiation. No Penicillium and Cladosporium spores survived after 40 and $80 \mathrm{~s}$ of IR heating, respectively. An approximate 4-log decrease in the survival was obtained for Aspergillus spores. Interestingly, the resistance of these three isolates (Penicillium, Aspergillus, and Cladosporium) to UV irradiation was considerably high. Moreover, the spores of Penicillium, Aspergillus, and Cladosporium were not completely sterilized even at $180 \mathrm{~s}$ of UV irradiation; only about a 3-log decrease of the initial population were found at $180 \mathrm{~s}$ of UV irradiation. On the other hand, Rhizopus spores were relatively sensitive to UV irradiation. Although the survival of Rhizopus spores was below the detectable level $(<2.4$ $\operatorname{logs}$ ), only $2 \operatorname{logs}$ of the initial populations were killed after $80 \mathrm{~s}$ of IR treatment. Byssochlamys spores were sensitive to both IR heating and UV irradiation, and did not survive after $80 \mathrm{~s}$ of IR heating and $60 \mathrm{~s}$ of UV irradiation.

Combination of IR heating with UV irradiation effectively inactivated the mold spores used in this study (Fig. 4). Both IR heating before UV irradiation (IR-UV treatment) and UV irradiation before IR heating (UV-IR treatment) considerable reduced the survival of the spores. At least $90 \%$ of the initial populations of viable mold spores was killed even at 5-s IR-UV. Combined treatment of 30-s IR-UV resulted in the reduction of more than $3 \mathrm{logs}$ of the initial population of any spore. Byssochlamys spores were especially inactivated 5 to 6 logs by the combined treatment of 5-s IR-UV, regardless of the treatment order. Moreover, 6 logs of Penicillium spores were also inactivated by 30 -s IR-UV. It is unclear whether the IR-UV treatment was more effective in spore inactivation than the UV-IR treatment, although IR-UV treatment was significantly effective $(p<0.05)$ in the inactivation of Penicillium spores after $20 \mathrm{~s}$ and Cladosporium spores after 10 s. When IR heating was simultaneously conducted with UV irradiation, the inactivation efficiency was almost the same as the sequential treatment (data not shown). Marquenie et al. (2002) reported the effect of combining UV-C irradiation with mild heat treatment (under $50^{\circ} \mathrm{C}$ ) on two kinds of mold conidia (Botrytis cinerea and Monilinia fructigena). They indicated that Botrytis spores were effectively inactivated when heat treatment was preceded by UV irradiation. However, the highest Monilinia spore inactivation was obtained by the reverse combined treatment. The inactivation efficiency depended on the kind of mold spore as suggested by Marquenie et al. (2002). Clarification of the killing mechanisms of mold spores by the combined treatment of IR heating with UV irradiation was important for application in various industrial fields. Inactivation by UV irradiation is postulated to be due to DNA damage, and that of IR heating may be caused by heat denaturation of essential proteins and destruction of the outer cell membrane. Therefore, inactivation of mold spores by combining IR treatment with UV irradiation could be synergistic.

\section{Conclusion}

Combination of IR heating with UV irradiation was considerably effective in inactivating various mold spores isolated from citrus fruits, compared with the single treatment. Thus, this combined treatment may have potential to kill any mold spore without over-heating, preventing quality degradation of the fruit products. However, it remains unclear whether treatment of IR heating followed by UV irradiation is more effective in spore inactivation than its reverse order. Further studies to elucidate the killing mechanisms (damage of cell wall, DNA and some essential factors of survival) are necessary for effective application of this combined treatment in the decontamination of the fruit surface.

Acknowledgements We thank Iwasaki Electric Co., Ltd., for providing the infrared halogen heater and ultraviolet lamp.

\section{References}

Aihara, M. and Lee, H.-J. (2005). Inspection of fungus (7) -Identification of fungus 3-. Bokin Bobai, 33, 373-377 (in Japanese).

Baka, M., Mercier, J., Corcuff, R., Castaingne, F., and Arul, J. (1999). Photochemical treatment to improve storability of fresh strawberries. J. Food Sci., 64, 1068-1072.

Hadjok, C., Mittal, G.S., and Warriner, K. (2008). Inactivation of human pathogens and spoilage bacteria on the surface and internalized within fresh produce by using a combination of ultraviolet light and hydrogen peroxide. J. Appl. Microbiol., 104, 1014-1024. Hamanaka, D., Uchino, T., Furuse, N., and Tanaka, S. (2005). Inactivation effect of infrared radiation heating on bacterial spores pretreated with various water activities. Biocontrol Sci., 10, 6165.

Hamanaka, D., Uchino, T., Furuse, N., Han, W., and Tanaka, S. (2006). Effect of the wavelength of infrared heaters on the inactivation of bacterial spores at various water activities. Int. J. Food Microbiol., 108, 281-285.

Jung, Y.J., Oh, B.S., and Kang, J.-W. (2008). Synergic effect of sequential or combined use of ozone and UV radiation for the disinfection of Bacillus subtilis spores. Water Res., 42, 1613-1621.

Kosuge, J. and Ota, T. (2005). Inspection of fungus (8) -Identification of fungus 4- Fungi imperfecti. Bokin Bobai, 33, 433-437 (in Japanese). 
Lee, H.-J. (2005). Inspection of fungus (6) -Identification of fungus 2-. Bokin Bobai, 33, 307-310 (in Japanese).

Marquenie, D., Lammertyn, J., Geeraerd, A.H., Soontijens, C., Van Impe, J.F., Nicolai, B.M., and Michiels, C.W. (2002). Inactivation of conidia of Botrytis cinerea and Monilinia fructigena using UV-C and heat treatment. Int. J. Food Microbiol., 74, 27-35.

Nigro, F., Ippolito, A., and Lima, G. (1998). Use of UV-C light to reduce Botrytis storage rot of table grapes. Postharvest Biol. Technol., 13, 171-181.

Song, J., Hildebrand, P.D., Fan, L., Forney, C.F., Renderos, W.E., and Campbell-Palmer, L. (2007). Effect of hexanal vapor on the growth of postharvest pathogens and fruit decay. J. Food Sci., 72, M108-M112.

Tournas, V.H. and Katsoudas, E. (2005). Mould and yeast flora in fresh berries, grapes and citrus fruits. Int. J. Food Microbiol., 105, 11-17.

Uchino, T., Inoue, A., Kawasaki, K., Hamanaka, D., and Tanaka, S.
(2004). The possibility of applying the infrared radiation to disinfection of fresh citrus fruit. J. Kyushu Branch Jpn Soc. Agric. Mach., 53, 7-10 (in Japanese with English abstract).

Valero, A., Begum, M., Leong, S.L., Hocking, A.D., Ramos, A.J., Sanchis, V., and Marin, S. (2007). Effect of germicidal UVC light on fungi isolated from grapes and raisins. Lett. Appl. Microbiol., 45, 238-243.

Yano, T. (2001). Sterilizing effect of sterilizing equipment using microwave combined with ultraviolet light. Bokin Bobai, 29, 91-98 (in Japanese).

Zhang, J. (2007). The potential of a new fungicide fludioxonil for stem-end rot and green mold control on Florida citrus fruit. Postharvest Biol. Technol., 46, 262-270.

Zheng, X., Tian, S., Gidley, M.J., Yue, H., and Li, B. (2007). Effects of exogenous oxalic acid on ripening and decay incidence in mango fruit during storage at room temperature. Postharvest Biol. Technol., 45, 281-284. 